Revista de Derecho YACHAQ

ISSN: 1817-597x (impresa) / ISSN: 2707-1197 (en linea)

Centro de Investigación de los Estudiantes de Derecho (CIED)

Universidad Nacional de San Antonio Abad del Cusco

N. ${ }^{\circ} 10-2019$

[pp. 63-72]

Fecha de recepción: 10/08/19

Fecha de aceptación:14/09/19

\title{
La Paisana Jacinta y los límites a la libertad de expresión en medios de comunicación masiva
}

\section{La Paisana Jacinta and Limits on Freedom of Speech in Mass Media}

\author{
Juan Carlos Ruiz Molleda ${ }^{[*]}$ \\ Álvaro Másquez Salvador ${ }^{[* *]}$
}

\begin{abstract}
RESUMEN: el presente artículo analiza la demanda de amparo interpuesta por ciudadanas cusqueñas contra el programa de televisión La Paisana Jacinta, así como la sentencia de primer grado emitida recientemente por el Poder Judicial. Bajo un enfoque constitucional e internacional, se cuestiona la caracterización pública de Jacinta en relación con las obligaciones de los medios de comunicación masiva en el Perú. Por último, se analizan los límites de la libertad de expresión y la censura previa, en aparente contradicción, en nuestra legislación y jurisprudencia.
\end{abstract}

ABSTRACT: this article analyzes the lawsuit filed by female citizens of Cusco against the television show La Paisana Jacinta, as well as the judgment issued recently by the Judiciary. Under a constitutional and international approach, the public characterization of Jacinta in relation to the obligations of mass media in Peru is questioned. Finally, we analyze the limits of freedom of expression and prior censorship, both in apparent contradiction, in our legislation and jurisprudence.

PALABRAS CLAVE: discriminación, derechos de la mujer, libertad de expresión, medios de comunicación masiva, censura previa.

KEY WORDS: discrimination, women's rights, freedom of Speech, mass media, prior restraint.

[*] Abogado por la Pontificia Universidad Católica del Perú, con postítulo en Derecho Procesal Constitucional y estudios concluidos en la Maestría de Derecho Constitucional en la misma casa de estudios. Es diplomado en Derechos Humanos y Derecho Internacional Humanitario por la American University (Estados Unidos) y en Derechos Económicos, Sociales y Culturales por la Universidad de los Andes (Colombia). Es coordinador del Área de Justicia Constitucional del Instituto de Defensa Legal y profesor en la Facultad de Ciencias Sociales de la Universidad Antonio Ruiz de Montoya. Contacto: jruiz@idl.org.p€

[**] Bachiller en Derecho por la Universidad de San Martín de Porres, con estudios complementarios en Sistema Interamericano de Derechos Humanos por el Institut International des Droits de l'Homme (Francia) y la Pontificia Universidad Católica del Perú, así como en Litigio Estratégico en Derechos Humanos por Open Society Justice Initiative y Hertie School of Governance (Alemania). Es especialista legal del Área de Justicia Constitucional del Instituto de Defensa Legal y asistente de docencia universitaria.

Contacto:amasquez@idl.org.pe 


\section{INTRODUCCIÓN}

En marzo de 2015, cuatro ciudadanas canchinas (Cecilia Paniura, Rosa Supho, Irene Quispe y Rosalinda Torres) interpusieron una demanda de amparo contra la emisión del programa de televisión La Paisana Jacinta. Las demandantes son mujeres indígenas, campesinas y quechuahablantes. Consideran que el personaje de Jacinta, que interpreta el actor Jorge Benavides, representa estereotipos negativos asociados a la mujer andina: violencia, vulgaridad, suciedad, falta de inteligencia y entre otros.

Latina, casa televisora de La Paisana Jacinta, comete en consecuencia un acto discriminatorio cuando emite el programa, a través de sus ondas de radio, a la colectividad. El caso fue recibido por el Juzgado Mixto de Wánchaq, en la ciudad del Cusco. La demanda fue declarada fundada casi cuatro años después, en noviembre de 2018.

El presente artículo, en dicho contexto, explica los alcances de la demanda planteada contra La Paisana Jacinta y la sentencia de primer grado, en especial con relación al presunto conflicto entre el derecho al honor y las libertades comunicativas, así como su relación con la prohibición de la censura previa.

\section{LA DEMANDA}

La demanda de amparo, como se dijo, fue interpuesta por cuatro ciudadanas cusqueñas en marzo de 2015, representantes de la Asociación por la Dignidad y Derechos de las Mujeres del Cusco, la Escuela de Mujeres Micaela Bastidas de Espinar y la Red de Mujeres de Canchis. Fue recibida por el Juzgado Mixto de Wánchaq y declarada improcedente in limine por razones de competencia geográfica. No obstante, esta decisión fue revocada en octubre de 2015 por la Primera Sala Civil del Cusco, que devolvió el caso al juez de primera instancia.

Como hechos lesivos, se identificó la emisión de los capítulos del programa de televisión por señal abierta e internet, así como la presentación del circo La Paisana Jacinta. Todo esto, se argumentó, violaba los derechos fundamentales de las deman- dantes a la igualdad y no discriminación, honor y buena reputación, identidad étnica y cultural, así como el deber de los medios de comunicación de colaborar con el Estado en la educación y formación moral y cultural y entre otros.

En el petitorio, se señala como pretensión principal lo siguiente:

Revisar y replantear el contenido del programa de televisión La Paisana Jacinta, a efectos de que sea respetuoso de los derechos de las mujeres indígenas andinas; es decir, que el humor vertido por el programa no descanse en la ridiculización, la burla, la discriminación y el racismo de estas [...].

Alternativamente, si Latina se rehusara a hacerlo, se planteó que el juez constitucional le ordene «ces[ar] la emisión del programa de televisión [...] hasta que haya sido reformulado".

\section{EL DERECHO AL HONOR Y LA REFORMU- LACIÓN DE JACINTA ${ }^{[1]}$}

Pero ¿a qué nos referimos con la reformulación de Jacinta? En principio, con realizar cambios en la caracterización del personaje -protagonista del programa de televisión- a fin de que no se asocie a estereotipos racistas. La vulgaridad, suciedad, falta de inteligencia y entre otras características a las que nos hemos referido deben dejar de ser representadas en Jacinta.

De lo contrario, se viola el derecho al honor de las demandantes, como ha sido reconocido por la jueza constitucional. Este derecho tiene dos dimensiones. En primer lugar, una dimensión externa referida a la protección de las posibilidades de participación de los individuos en las relaciones sociales frente a las alteraciones que pudieran derivarse de las conductas llevadas a cabo por terceros. Esto nos remite al concepto de heteroestima, el cual puede ser entendido como la capacidad de aparecer ante los demás en condiciones de semejanza, lo que permite, precisamente, la participación en los sistemas sociales.

[1] Al respecto, véase: Másquez Salvador y Ruiz Molleda (2017). 
Como señala Montoya Chávez (2008), es importante que la actividad o comportamiento del individuo, entendiendo a este como medio de intervención en la sociedad y como fuente de expectativas de reconocimiento frente a los demás, no se vea falseado, distorsionado o alterado. En otras palabras, es necesario garantizar las condiciones que permiten a cualquier individuo el reconocimiento social.

Por otro lado, una dimensión interna, constituida por las pretensiones mínimas de respeto que emanan de la persona por el mero hecho de serlo. Nuevamente, siguiendo a Montoya Chávez (2008), diríamos que el fundamento de esta dimensión es el honor. En tal sentido, su bien jurídico tutelado será «la competencia y suficiencia de la persona de estar y actuar en una porción identificable de la sociedad como parte integrante de ella y siendo reconocida como tal». La dignidad constituye un espacio infranqueable frente a cualquiera, puesto que todo ataque a ella lleva consigo la negación de la misma como integrante de la especie humana (Ruiz, 2017).

El Tribunal Constitucional ha recogido esta postura. Con precisión, ha señalado:

Se puede considerar que el honor, sobre la base de la dignidad humana, es la capacidad de aparecer ante los demás en condiciones de semejanza, lo que permite la participación en los sistemas sociales y corresponde ser establecido por la persona en su libre determinación (STC. Exp. N. ${ }^{\circ}$ 3362-2004-AA/TC, 2006, fundamento 14b).

Así, la caracterización de Jacinta en televisión abierta menoscaba hondamente la capacidad de las mujeres andinas, campesinas y quechuahablantes, de aparecer ante los demás en condiciones de semejanza. Por el contrario, la transmisión de Frecuencia Latina permite que su presencia continúe siendo ligada a un conjunto de estereotipos negativos. Esto dificulta su capacidad de integración a la sociedad. Alimenta, en consecuencia, la negación de la propia identidad y la estigmatización de los pueblos indígenas. Los impactos en niños, niñas y adolescentes son aún mayores y más intensos, pues los hace susceptibles de sufrir acoso escolar.

En este sentido, la emisión de La Paisana Jacinta viola doblemente el derecho al honor de las mujeres campesinas indígenas, y las demandantes en particular. En primer lugar, por ser una ofensa hacia ellas y, en segundo lugar, por generarles un grave desprestigio social frente a los demás, una desacreditación que compromete de forma determinante su vida y su participación en la comunidad y en la sociedad.

\section{LIBERTADES COMUNICATIVAS Y CENSURA PREVIA}

El problema jurídico no es otro que la colisión entre la libertad de opinión y el derecho al honor. O, como diríamos en expresiones más precisos, la colisión entre los derechos de las personas (honor) y los derechos de comunicación del discurso o libertades comunicativas (expresión e información). Para resolver el problema es primero necesario estudiar los alcances de las libertades comunicativas.

Según el artículo 2.4 de la Constitución, toda persona tiene derecho a «las libertades de información, opinión, expresión y difusión del pensamiento mediante la palabra oral o escrita o la imagen, por cualquier medio de comunicación social». No obstante, este derecho no es absoluto. No otorga a su titular facultades ilimitadas para emitir cualquier clase de opinión o en cualesquiera circunstancias. Su contenido constitucional se delimita en parte por el derecho contenido en el artículo 2.7 de la misma Constitución, que reconoce que toda persona tiene derecho al honor y la buena reputación.

Las libertades comunicativas, debemos precisar, se reconducen a dos derechos: la libertad de información, que es la trasmisión de información objetiva, la cual puede ser verdadera o falsa y, de otro lado, la libertad de opinión o expresión, referida a la trasmisión de opiniones y apreciaciones de carácter subjetivo. En este caso, el límite lo representa el respeto al honor y la dignidad de las personas.

En efecto, la dignidad de la persona es el fundamento de los derechos fundamentales, siendo estas genuinas expresiones del libre desarrollo de la personalidad que perfilan su contenido material. En consecuencia, es el libre desarrollo de la personalidad lo que debe ser protegido. Es esto lo que se ve comprometido cuando se afecta el derecho al honor de las mujeres indígenas quechuahablantes. 
Debemos ser enfáticos en señalar que la libertad de expresión no ampara el derecho al insulto. Es necesario reiterar que los derechos fundamentales no son absolutos. Su ejercicio está limitado por su propio contenido y por su relación con otros bienes constitucionales y su protección (STC. Exp. N. ${ }^{\circ}$ 05975-2008-AA/TC, 2010, fundamento 7). En este sentido, estos solo podrán ser restringidos en base a criterios de razonabilidad y proporcionalidad (STC. Exp. N. ${ }^{\circ}$ 02700-2006-PHC/TC, 2007, fundamento 18); por lo que es posible afirmar que también la libertad de expresión no es irrestricta.

El Derecho Internacional de los Derechos Humanos se ha referido a estos límites o restricciones. El Pacto Internacional de Derechos Civiles y Políticos y la Convención Americana sobre Derechos Humanos establecen, para el caso del derecho a la información, en el artículo 19 ${ }^{[2]}$, que «entraña deberes y responsabilidades especiales, por lo que está sujeto a una restricción como es la de asegurar [...] el respeto a los derechos o reputación de los demás».

Sin embargo, está prohibida la censura previa. Así lo establece la Constitución Política en su artículo 2.4: «sin previa autorización ni censura ni impedimento alguno» y la Convención Americana de Derechos Humanos, en su artículo 13, «no puede estar sujeto a previa censura sino a responsabilidades ulteriores».

Al respecto, el TC señaló en el caso Caja Rural de Ahorro y Crédito de San Martín (STC. Exp. N. ${ }^{\circ}$ 00905-2001-AA/TC, 2002), donde los demandantes pretendían que una emisora radial se abstenga de difundir noticias falsas, lo siguiente:

La pretensión formulada por la demandante, en el sentido de que se expida una orden judicial en virtud de la cual se impida que los emplazados pueden seguir difundiendo hechos noticiosos, es incompatible con el mandato constitucional que prohíbe que se pueda establecer al ejercicio de la libertad de información y expresión, censura o impedimentos alguno (fundamento 15).

Sin embargo, añade el Tribunal que no es posible desproteger los derechos al honor o a la buena reputación por causa del ejercicio arbitrario del derecho a la información, por lo que «en tales casos, el propio ordenamiento constitucional ha previsto que sus mecanismos de control tengan que actuar en forma reparadora, mediante diversos procesos que allí se tiene previstos» (STC. Exp. N. ${ }^{\circ}$ 00905-2001$A A / T C, 2002)$.

Esta posición también ha sido asumida por la Corte Interamericana de Derechos Humanos cuando estipula que:

En primer lugar, la prohibición de la censura previa la cual es siempre incompatible con la plena vigencia de los derechos enumerados por el artículo 13, salvo las excepciones contempladas en el inciso 4 referentes a espectáculos públicos, incluso, si se trata supuestamente de prevenir por ese medio un abuso eventual de la libertad de expresión. En esta materia, toda medida preventiva significa, inevitablemente, el menoscabo de la libertad garantizada por la Convención. (Corte IDH. Opinión Consultiva 5/85, 1985, p. 38)

Esta posición ha sido reiterada en la sentencia del caso La Última Tentación de Cristo (Olmedo Bustos y otros) contra Chile, a propósito de la censura judicial impuesta a una película por presuntamente afectar valores cristianos.

\section{INTERPRETACIÓN CONSTITUCIONAL DE LA PROHIBICIÓN}

Lo cuestionable en el caso de La Paisana Jacinta, según una interpretación literal de la prohibición de la censura previa, es que no existen mecanismos efectivos para proteger el honor de las mujeres indígenas campesinas. Incluso, cuando se trata de la violación masiva de un derecho fundamental - por la amplia cobertura que alcanza un programa de televisión- de la que se tiene total certeza, aun antes de la transmisión de un nuevo capítulo.

En otras palabras, lo que sostiene esta interpretación es que derechos como el honor y la buena reputación podrán ser violados. Al admitir la relevancia de las libertades comunicativas por sobre el

[2] Pacto Internacional de Derechos Civiles y Políticos de fecha 16 de diciembre de 1966. 
derecho al honor y a la buena reputación, en principio se reconoce que cuando se dé este supuesto de colisión, los jueces constitucionales están incapacitados de impedir que se configure esa violación (Castillo, 2006, p. 120).

Aceptar la relevancia de las libertades comunicativas, mediante esta interpretación cuestionable de la disposición que contiene la prohibición de censura previa, convierte a las libertades comunicativas en la práctica en absolutas o, cuanto menos, preponderantes. En tal escenario, el afectado en su honor deberá conformarse con activar mecanismos ex post de sanción o reparación.

A juicio de Castillo Córdova (2006), las libertades comunicativas se muestran como «una suerte de súper libertad indestructible e inexpugnable que avasalla a aquellas otras libertades o derechos que tienen la infortuna de cruzarse por los espacios que ella decide andar». La consecuencia no puede ser otra, señala: «[se] condena al demandante afectado en su derecho al honor a aceptar una posible vulneración de su derecho al honor o buena reputación derivándolo a que eventualmente active los mecanismos reparadores del hecho» (p. 120).

El profesor peruano Castillo (2006) se refiere así a esta interpretación:

Habrá casos en los que se ha de asumir a la Constitución no como una unidad sistemática, sino como una realidad normativa contradictoria. Y eso será la Constitución si se acepta que la Constitución dispone la protección del derecho al honor solo en determinados supuestos: en aquellos en los que no concurra ni la libertad de expresión, ni la libertad de información. Y eso será la Constitución si aceptamos que la cláusula constitucional de censura previa exige admitir que la norma que reconoce el derecho al honor pierde toda virtualidad jurídica cuando nos hallemos frente al ejercicio de una libertad comunicativa $[. .$.$] . Poco importa que la información sea$ falsa, incluso poco importa el ánimus del informador, o el lenguaje injurioso o no, que es empleado para acompañar la información que se trasmite (p. 121).
Sin embargo, es necesario reinterpretar, desde el Derecho Constitucional, lo que entendemos por censura previa y sus alcances. Para empezar, porque los mecanismos reparadores del derecho al honor — de carácter ulterior — no son realmente eficaces. Se plantea, en la práctica, por lo menos, dos mecanismos reparadores: la rectificación y la acción indemnizatoria; incluso, también, se habla de mecanismos sancionadores, por medio del Derecho Penal.

Descartamos de plano esta última opción, pues que los derechos fundamentales deben ser protegidos mediante acciones de naturaleza restitutiva, como las constitucionales, antes que estrictamente punitivas, como las penales.

En relación con la rectificación, se trata de un derecho reconocido en el artículo 2.7 de la Constitución Política que protege, de manera limitada, el derecho al honor, "contra el escarnecimiento o la humillación, ante sí o ante los demás» (Exp. N. ${ }^{\circ}$ 00446-2002-AA/TC, 2003, fundamento 2). La rectificación, sin embargo, no neutraliza ni regresa las cosas al estado anterior a la violación del derecho al honor.

Castillo Córdova (2006) señala:

Podría darse [la restitución del derecho] solo en el supuesto que la rectificación pueda ser igualmente vista u oída por todos aquellos que vieron u oyeron la trasmisión de hechos falsos, de modo que estos adquirieran el convencimiento de que un sujeto no es o no ha hecho lo que inicialmente se dijo que era o que había hecho (p. 125).

Toller (1999) señala, en tal contexto, que el derecho al honor es irreparable «porque las difamaciones que se han ido propalando son casi imposibles de retirar, del mismo modo que lo es recoger todas las plumas que se han ido arrojando a lo largo de una ciudad en un día de viento» (p. 186).

Por otro lado, la acción indemnizatoria tampoco restaura el derecho afectado. No resulta realmente reparadora, pues nos encontramos ante un daño material, sino moral, que no es posible de reparar mediante dinero. 
Toller (1999) indica:

Ante este tipo de daño no parece que el afectado pueda encontrar la satisfacción adecuada - justa compensación - en una indemnización económica. La razón de ello es que un daño moral no puede resarcirse pecuniariamente en estricta justicia, esto es, en virtud de la obligación de devolver exactamente lo que se recibió, o de restaurar la cosa tal como estaba antes de que se le hubiese dañado (p. 167).

Frente al problema, una interpretación constitucional de la noción de censura previa debe corregir las incongruencias de la Constitución y el propio Derecho Internacional al preferir ex ante la protección de las libertades comunicativas frente al honor. Para esto, debemos recurrir a los principios de la interpretación constitucional, en particular al de unidad de la Constitución.

Castillo Córdova (2006) señala que ambos derechos deben interpretarse de manera coordinada, de tal manera que se evite cualquier contradicción entre sí. De tal forma, señala:

El principio de unidad y sistematicidad de la norma constitucional no permite interpretar la cláusula de la prohibición de consulta previa como un instrumento que no permita evitar vulneraciones al derecho al honor o a la intimidad; o, con otras palabras, como instrumento que permita hacer de las libertades comunicativas libertades prácticamente absolutas e ilimitadas. Así, se permitiría evitar que se difunda información que vulneraría derechos como el honor.

En el presente caso, esto implica que se debe optar por una interpretación de los artículos 2.4 y 2.7 de la Constitución, que proteja igualmente las libertades comunicativas y el derecho al honor, dado su igual valor normativo. La cobertura de esta interpretación está en el artículo 200.2 de la Constitución, que reconoce la procedencia de la demanda de amparo por la afectación del derecho al honor y entre otros derechos. Del mismo modo, el artículo 2 del Código Procesal Constitucional prevé que el amparo procede en caso de que la amenaza de violación de un derecho constitucional sea cierta y de inminente realización.
Se permite, de tal forma, «abrir una de las puertas procesales posibles de cruzar a fin de lograr que, preventivamente, cuando haya la certeza de que una información de trasmitirse viole el derecho al honor o a la intimidad, se active el andamiaje procesal constitucional a fin de lograr que esa amenaza de vulneración no llegue a convertirse en violación efectiva». En esa línea, es necesario preguntarnos si la amenaza cierta e inminente de vulneración del derecho al honor como consecuencia de la difusión de La Paisana Jacinta, ise violaría la prohibición de censura previa si un juez constitucional ordenase la no emisión del programa? ¿Qué debemos entender entonces por censura previa?

Si se revisa con detenimiento el artículo 2.4 de la Constitución Política, se advertirá que esta precisa «sin previa autorización ni censura ni impedimento alguno", y el artículo 13.2 de la Convención Americana prevé que «no puede estar sujeto a previa censura, sino a responsabilidades ulteriores». A juicio de Castillo Córdova (2006), el mandato constitucional no se dirige al órgano judicial, sino a la entidad administrativo, pues la razón de ser de la censura previa «es evitar que el poder político pueda intervenir para callar a un medio de comunicación crítico con su actuación y que como tal le resulte incómodo», y «nunca fue permitir violaciones de derechos constitucionales o hacer ineficaces los mecanismos de control jurídico frente a situaciones de amenaza cierta e inminente de derechos constitucionales a través de la difusión de información» (p. 130).

A nivel interno, añade Montoya Chávez (2010), se ha sostenido que:

El ámbito de la censura previa se centra en los ámbitos administrativo, político o económico. Sin embargo, la situación cambia totalmente cuando el examen que se deba realizar es netamente judicial, y ya no se inserta en tales espectros. La censura no puede ser lo suficientemente amplia como para incorporar una negativa a la judicatura a intervenir en estos supuestos, más aún si el propio ordenamiento jurídico prevé formas de protección preventiva, específicamente, cuando se trata de derechos fundamentales (p. 139). 
La consecuencia es evidente. No puede prohibirse que un juez constitucional conozca una demanda de amparo por amenaza cierta e inminente de violación del derecho al honor, tampoco que actúe preventivamente en salvaguarda del derecho. Así, señala Castillo Córdova (2006):

No puede descartarse una actuación preventiva por parte de la autoridad judicial y eventualmente por el mismo Tribunal Constitucional. Precisamente, esa actuación preventiva es exigida para lograr una más plena eficacia y garantía de todos los derechos constitucionales $[\ldots]$, hay que encontrar medidas eficaces que empeladas razonablemente en cada caso concreto no vayan a suponer una restricción o violación de las libertades comunicativas mismas. (p. 129)

Montoya Chávez (2010), citando a Toller, precisa que aunque esta alternativa implica grandes riesgos «merece ser seguida por ofrecer la única salida posible en situaciones donde, si se denegara la tutela, se cometerá una notoria injusticia a la vista del juez, que se convertiría en un espectador privilegiado de la realización inexorable de un daño grave e irreparable a derechos fundamentales y bienes públicos» (p. 141).

A juicio de Montoya Chávez, habrá que interpretar los artículos 2.4 y 2.7 de la Constitución, así como el 13 de la Convención Americana, en el sentido que:

Toda persona ejercitará sus derechos a la expresión y a la información sin previa autorización, ni censura ni impedimentos algunos, salvo que exista un hecho u omisión por parte de cualquier autoridad, funcionario o persona, que amenace los demás derechos reconocidos en la Constitución, dentro de los cuales, lógicamente, se encontrará los de respeto propio.

El tal sentido, añade Montoya Chávez (2010) que el control judicial está plenamente justificado «si es que medida la salvaguardia de otro derecho fundamental: si se sabe que el titular de un derecho va a ejercerlo abusivamente, el ordenamiento no puede permitir que, a través de este, se afecte otro» (p. 139).
Consecuentemente, consideramos que es posible realizar una labor de control judicial previa en los casos donde se tenga certeza de la afectación del derecho al honor. El juez constitucional, en tales casos, tendrá la posibilidad de examinar cada situación en concreto y ofrecer una respuesta, positiva o negativa, en base a la protección de los derechos de las personas.

\section{VI. ¿CONFLICTO DE DERECHOS FUNDA- MENTALES?}

Corresponde ahora preguntarnos: ¿existe o no un conflicto de derechos fundamentales en el caso de La Paisana Jacinta? En apariencia, no, pues nos encontramos frente a un ejercicio desproporcionado y abusivo de un derecho fundamental (las libertades comunicativas) frente a la afectación de otro (honor). Es decir, no existiría conflicto porque no es parte contenido constitucionalmente protegido de las libertades comunicativas el derecho a insultar a las personas, mucho menos a un sector importante de la población nacional, como representan las mujeres indígenas quechuablantes.

Siendo así, el juez constitucional está facultado para requerir a Frecuencia Latina la reformulación del personaje de Jacinta o su retiro del programa de televisión, a través de un control judicial respetuoso de la prohibición de la censura previa.

Ciertamente, no resulta adecuado referirnos a la existencia de un conflicto. Sin embargo, es importante señalar: ¿aun si existiera un conflicto de derechos fundamentales, qué derecho debiera ser preferido en el caso concreto? Al respecto, nos valemos del test de proporcionalidad como herramienta para examinar la constitucionalidad de las medidas que posiblemente afectan derechos fundamentales.

En el presente caso, analizaremos la constitucionalidad del programa de televisión, siendo constitucional y válido solo si este representa una limitación o afectación idónea, necesaria y ponderada de derechos fundamentales.

El análisis de idoneidad comprende el examen de si la medida es idónea para la protección de otros derechos y bienes constitucionales. En el presente caso, si bien el programa se inscribe en el marco del ejercicio de las libertades comunicati- 
vas, sus contenidos no persiguen ninguna finalidad constitucional. Es decir, no concretan derechos o bienes jurídico-constitucionales. Por el contrario, lo único que se hace es pretender el entretenimiento sobre la base a la discriminación.

Por otro lado, conforme al análisis de necesidad, la medida será constitucional solo si no existe otra medida alternativa que restrinja en menor grado derechos fundamentales. En este caso, es evidente que existen muchas otras maneras de promover el entretenimiento sin tener que recurrir a la generación de estereotipos que promueven el racismo las mujeres indígenas.

Por último, la medida será ponderada o proporcional en sentido estricto solo si se demuestra que la intensidad de la afectación de estos derechos es menos gravosa en comparación con la intensidad de afectación que sufriría el derecho o bien constitucional que promueva la medida a implementarse; de lo contrario, la medida deberá ser prohibida y excluida también su implementación.

Al respeto, es claro que debe preferirse el derecho al honor por encima de las libertades comunicativas en el caso concreto. Ello debe protegerse, especialmente, de aquellos derechos cuyo grado de reparabilidad sea más difícil, complejo o imposible, como resulta ser el honor y la prohibición de discriminación por origen étnico. En este sentido, el daño moral - que se concreta con la violación del derecho al honor- no es reparable, mientras que las libertades comunicativas, en un contexto como el presente, sí lo son.

Cabe señalar que nos encontramos frente a uno de aquellos casos en los que no es posible armonizar los bienes jurídicos en conflicto. La reformulación de Jacinta, en consecuencia, no representa una opción arbitraria, sino que esta es consecuencia de la importancia del derecho al honor, por estar en conexión más directa con la dignidad humana y del derecho a la igualdad, y por carecer el programa de televisión de contenido público o interés público. Como señala Grández Castro (2010), la ponderación en derechos indirectamente vinculados a la dignidad humana, como las libertades económicas, puede considerarse fácil. Agrega que la ponderación, en tales supuestos, "confirma una suerte de mayor "peso abstracto" que representan algunos derechos que, si bien formalmente valen lo mismo desde la Constitución, no obstante, reciben diferente valoración de cara a un caso concreto» (pp. 212-213).

En esta misma línea, se deberá preferir la protección de los derechos cuya violación tenga una mayor durabilidad en el tiempo. En el presente caso, los impactos negativos de La Paisana Jacinta son de mayor durabilidad, toda vez que fomenta estereotipos que refuerzan patrones de conducta racistas, históricamente presentes en el país. Las afectaciones al honor, en consecuencia, tardarían mucho en repararse, si esto fuera posible. Las consecuencias escapan a las mujeres indígenas quechuablantes y alcanzan, acto seguido, a la colectividad nacional.

En conclusión, de conformidad con lo señalado por Grández Castro (2010), podemos concluir que la intensidad de los derechos intervenidos es grave, toda vez que nos encontramos ante una afectación bastante severa del derecho al honor. No de una persona o dos, sino de un sector importante de la población peruana. Por el contrario, el grado de realización de las libertades comunicativas es leve, toda vez que no basta con la reformulación de Jacinta para asegurar la continuidad del programa de televisión, siendo la cancelación la última opción.

En otras palabras, los derechos afectados por La Paisana Jacinta (honor, igualdad, dignidad, identidad étnica y entre otros) son de mayor importancia que los derechos que este pretende satisfacer (libertades comunicativas y económicas). En consecuencia, no tratándose de una limitación o restricción idónea, necesaria y ponderada, la emisión del programa de televisión no satisface el principio de proporcionalidad y, por tanto, deberá ser declarado inconstitucional.

\section{LA SENTENCIA JUDICIAL}

Precisamente, la sentencia judicial en el presente caso reconoció la violación de derechos fundamentales, especialmente, los de igualdad y no discriminación por razón de origen étnico, honor e identidad cultural. Para esto, se tomaron en cuenta notas descriptivas de algunos episodios del programa de televisión, ofrecidas por las demandantes, y las apreciaciones críticas del Comité para la Elimi- 
nación de la Discriminación Racial en una sesión pública, en agosto de 2014.

No obstante, el juzgado consideró que la transmisión del programa de televisión cesó en marzo de 2015 - hecho con el que discrepamos-y, por tanto, había cesado la afectación de los derechos de las demandantes.

Aun así, en razón de la naturaleza innovativa del amparo y los derechos fundamentales, ordenó a Frecuencia Latina «no volver a incurrir en las acciones materia de la presente demanda», así como retirar los vídeos contenidos en el canal de YouTube de la casa televisora.

\section{CONCLUSIONES}

En el presente caso, como ha sido reconocido por el Juzgado Mixto de Wánchaq en una sentencia estimatoria, el programa de televisión La Paisana Jacinta viola derechos fundamentales, al asociar a la mujer indígena, campesina y quechuahablante con estereotipos racistas (como la violencia, vulgaridad, suciedad y falta de inteligencia), que tanto daño han hecho el país durante los últimos siglos.

En ese sentido, se viola el derecho al honor de las demandantes, en sus dimensiones interna y externa, mediante el uso desproporcionado y abusivo de las libertades comunicativas. Tal uso no es amparado por el Derecho. Para empezar, porque las libertades comunicativas no incluyen en su contenido constitucionalmente protegido el derecho al insulto.

No existe un conflicto de derechos, en consecuencia. Sin embargo, aunque lo hubiera, es posible afirmar que la transmisión del programa de televisión no satisface el principio de proporcionalidad: por no perseguir ningún derecho o bien jurídico fundamental, por existir otras maneras no discriminatorias de promover el entretenimiento, y por el daño irreparable y extendido en el tiempo que generan, mucho mayor que los intereses comunicacionales o económicos que el programa de televisión pretende satisfacer.

La reformulación del personaje de Jacinta, de tal forma que se supriman los estereotipos negativos que representa, se constituye como alternativa para salvaguardar los derechos de las mujeres indígenas y evitar la cancelación del programa de televisión, como ha sido ordenado por el juzgado.

Cabe señalar que la prohibición de la censura no puede entenderse, en especial en casos como el presente, como una regla absoluta. Es necesario interpretarla desde el Derecho Constitucional. Por consiguiente, cuando se acrediten situaciones de amenaza cierta e inminente de derechos fundamentales como el honor, deberá ser posible someter contenidos al control judicial —no administrativopara su evaluación en base a criterios objetivos.

\section{BIBLIOGRAFÍA}

Castillo Córdova, L. (2006). Las libertades de expresión e información. Lima: Palestra.

Chávez Montoya, V. H. (2008). El honor frente a la expresión y a la información. Un conflicto que quiso ser resuelto por la Corte Suprema. Comentarios a los precedentes vinculantes en materia penal de la Corte Suprema. pp. 677-729.

Corte IDH. Opinión Consultiva 5/85, Colegiatura obligatoria de los periodistas (Corte Interamericana de Derechos Humanos, 13 de noviembre de 1985).

Grández Castro, P. (2010). Tribunal Constitucional y argumentación jurídica. Lima: Palestra.

Másquez Salvador, Á., \& Ruiz Molleda, J. C. (21 de julio de 2017). Justicia Viva. Recuperado de: http://www.justiciaviva.org.pe/new/la-reformulacion-de-la-paisana-jacinta/?fbclid = IwAR1jy3W 5oMKYel7Aj2Jz33hQmsOU9o9tQs8IpgbQGCG

Montoya Chávez, V. (2010). La censura previa judicializada. Actualidad Jurídica (134).

Ruiz Molleda, J. C. (2017). Justicia Viva. En Los límites de la libertad de opinión. A propósito de la acusación de Martha Chávez a Gisela Ortiz de ser terrorista. Recuperado de: https://goo. $\mathrm{gl} / \mathrm{GqSn} 1 \mathrm{~b}$

STC. Exp. N. ${ }^{\circ}$ 00446-2002-AA/TC, STC. Exp. N. ${ }^{\circ}$ 00446-2002-AA/TC, fund. 2 (Tribunal Constitucional, 19 de diciembre de 2003).

STC. Exp. N. ${ }^{\circ}$ 00905-2001-AA/TC, STC. Exp. N. ${ }^{\circ}$ 00905-2001-AA/TC (Tribunal Constitucional, 14 de agosto de 2002). 
STC. Exp. N. ${ }^{\circ}$ 02700-2006-PHC/TC, STC. Exp. N. ${ }^{\circ}$ 02700-2006-PHC/TC (Tribunal Constitucional, 23 de marzo de 2007).

STC. Exp. N. ${ }^{\circ}$ 05975-2008-AA/TC, STC. Exp. N. ${ }^{\circ}$ 05975-2008-AA/TC (Tribunal Constitucional, 12 de mayo de 2010).
STC. Exp. N. ${ }^{\circ} 3362-2004-A A / T C$, STC. Exp. N. ${ }^{\circ}$ 3362-2004-AA/TC (Tribunal Constitucional, 29 de agosto de 2006).

Toller, F. (1999). Libertad de prensa y tutela judicial efectiva. Estudio de la prevención judicial de daños derivados de informaciones. Buenos Aires: La Ley. 